\title{
Existence theorems for relaxed $\eta-\alpha$ pseudomonotone and strictly $\eta$-quasimonotone generalized variational-like inequalities
}

\author{
Areerat Arunchai ${ }^{1}$, Somyot Plubtieng ${ }^{1 *}$ and Ching-Feng Wen ${ }^{2}$
}

"Correspondence:

Somyotp@nu.ac.th

'Department of Mathematics,

Faculty of Science, Naresuan

University, Phitsanulok, 65000,

Thailand

Full list of author information is

available at the end of the article

\begin{abstract}
In this paper, we prove the existence of solutions for a variational-like inequality and a generalized variational-like inequality in the relaxed $\eta-\alpha$ pseudomonotone and strictly $\eta$-quasimonotone cases in Banach spaces by using the KKM technique. The results presented in this paper improve and extend some corresponding results of several authors.
\end{abstract}

Keywords: variational-like inequality; generalized variational-like inequality; relaxed $\eta$ - $\alpha$ pseudomonotone operator; strictly $\eta$-quasimonotone operator; solution existence

\section{Introduction}

The variational inequality was first introduced and studied in the finite-dimensional Euclidean space by Giannessi [1]. Variational inequality problems play a critical role in many fields of science, engineering, and economics. In the last four decades, since the time of the celebrated Hartman-Stampacchia theorem (see $[2,3])$, the existence of a solution of a variational inequality, a generalized variational inequality, and other related problems has become a basic research topic which continues to attract the attention of researchers in applied mathematics (see for instance [4-13], and the references therein).

In 1995, Chang et al. [14] introduced and studied the problem of the existence of solutions and the perturbation problem for some kind of variational inequalities with monotone and semimonotone mappings in nonreflexive Banach spaces. Recently, Verma [15] studied a class variational inequality relaxed monotone mapping. Moreover, Fang and Huang [16] obtained the existence of solutions for variational-like inequalities with relaxed $\eta-\alpha$ monotone mappings in reflexive Banach spaces. In 2003, Facchinei and Pang $[17,18]$ used the degree theory to obtain a necessary and sufficient condition of variational inequality problems for continuous pseudomonotone mappings in a finite-dimensional space. In 2008, Kien et al. [19] proposed some extensions of the results of Facchinei and Pang $[17,18]$ to the case of variational inequalities and generalized variational inequalities in infinite-dimensional reflexive Banach spaces.

On the other hand, Bai et al. [20] introduced the new concept of relaxed $\eta-\alpha$ pseudomonotone mappings. By using the KKM technique, they obtain some existence results

o2014 Arunchai et al.: licensee Springer. This is an Open Access article distributed under the terms of the Creative Commons Attribution License (http://creativecommons.org/licenses/by/2.0), which permits unrestricted use, distribution, and reproduction in any medium, provided the original work is properly cited. 
for variational-like inequalities with relaxed $\eta-\alpha$ pseudomonotone mappings in reflexive Banach spaces. In 2007, Wu and Huang [21] introduced the two new concepts of relaxed $\eta-\alpha$ pseudomonotonicity and relaxed $\eta-\alpha$ demipseudomonotonicity in Banach spaces. In 2009, Pourbarat and Abbasi [22] tried to replace some conditions of the work of Wu and Huang [21] with some new conditions. Moreover, they present the solvability of variational-like inequalities with relaxed $\eta-\alpha$ monotone mappings in arbitrary Banach spaces (see also in $[2,15-20]$ and [23-28]).

Inspired and motivated by [19], we introduce a new definition of relaxed $\eta-\alpha$ pseudomonotone mappings and prove the existence of solutions for variational-like inequality and generalized variational-like inequality with relaxed $\eta$ - $\alpha$ pseudomonotone mappings and strictly $\eta$-quasimonotone mappings in Banach spaces by using KKM technique. The results presented in this paper improve and extend some corresponding results of several authors.

\section{Preliminaries}

Let $X$ be a real reflexive Banach space with dual space $X^{*}$ and $\langle\cdot, \cdot\rangle$ denoted the pairing between $X^{*}$ and $X$. Let $K$ be a nonempty subset of $X$, and $2^{X}$ denote the family of all the nonempty subset of $X$ and $\Phi: K \rightarrow 2^{X^{*}}$ and $\eta: K \times K \rightarrow X$ be mappings. The generalized variational-like inequality defined by $K$ and $\Phi$, denoted by $\operatorname{GVLI}(K, \Phi)$, is the problem of finding a point $x \in K$ such that

$$
\exists x^{*} \in \Phi(x), \quad\left\langle x^{*}, \eta(y, x)\right\rangle \geq 0 \quad \forall y \in K .
$$

The set of all $x \in K$ satisfying (2.1) is denoted by $\operatorname{SOL}(K, \Phi)$. If $\Phi(x)=\{F(x)\}$ for all $x \in K$, where $F: K \rightarrow X^{*}$ is a single-valued mapping, then the problem $\operatorname{GVLI}(K, \Phi)$ is called a variational-like inequality and the abbreviation $\operatorname{VLI}(K, F)$ is the problem of finding an $x \in K$ such that

$$
\langle F(x), \eta(y, x)\rangle \geq 0 \quad \forall y \in K .
$$

We introduce the definition of relaxed $\eta-\alpha$ pseudomonotone for $\alpha$ mapping which comes from a family of functions which contains all mappings $\alpha$ given in [20]. In fact, the new definition is an extension of Definition 2.1 in [20]. Then we recall some definitions and results which are needed in the sequel.

We introduce the family

$$
\mathbf{A}=\left\{\alpha: \alpha: X \rightarrow \mathbb{R} ; \limsup _{t \rightarrow 0^{+}} \frac{\alpha(t \eta(x, y))}{t}=0 \forall(x, y) \in K \times K\right\} .
$$

We note that if $\alpha(t x)=k(t) \alpha(x)$, for all $x \in X$ where $k$ is a function from $(0, \infty)$ to $(0, \infty)$ with $\lim _{t \rightarrow 0} \frac{k(t)}{t}=0$, then $\alpha \in A$.

Definition 2.1 The mapping $F: K \rightarrow X^{*}$ is said to be:

(i) Relaxed $\eta$ - $\alpha$ pseudomonotone if there exist $\eta: K \times K \rightarrow X$ and $\alpha: X \rightarrow \mathbb{R}$ with $\alpha \in A$, such that for every distinct points $x, y \in K$,

$$
\langle F(y), \eta(x, y)| \geq 0 \quad \Rightarrow \quad\langle F(x), \eta(x, y)| \geq \alpha(\eta(x, y)) .
$$


If $\eta(x, y)=x-y$ for all distinct points $x, y$ in $K$, then (2.3) becomes

$$
\langle F(y), x-y\rangle \geq 0 \quad \Rightarrow \quad\langle F(x), x-y| \geq \alpha(x-y)
$$

and $F$ is said to be relaxed $\alpha$ pseudomonotone.

(ii) Strictly $\eta$-quasimonotone if there exist $\eta: K \times K \rightarrow X$ such that for every distinct points $x, y \in K$,

$$
\langle F(y), \eta(x, y)\rangle>0 \Rightarrow\langle F(x), \eta(x, y)\rangle>0 .
$$

If $\eta(x, y)=x-y$ for all distinct points $x, y$ in $K$, then (2.4) becomes

$$
\langle F(y), x-y\rangle>0 \quad \Rightarrow \quad\langle F(x), x-y\rangle>0,
$$

and $F$ is said to be strictly quasimonotone.

Definition 2.2 The mapping $\Phi: K \rightarrow 2^{X^{*}}$ is said to be:

(i) Relaxed $\eta$ - $\alpha$ pseudomonotone if there exist $\eta: K \times K \rightarrow X$ and $\alpha: X \rightarrow \mathbb{R}$ with $\alpha \in A$,

$$
\begin{aligned}
& \left\langle y^{*}, \eta(x, y)\right| \geq 0, \quad \exists y^{*} \in \Phi(y) \\
& \quad \Rightarrow \quad\left\langle x^{*}, \eta(x, y)\right\rangle \geq \alpha(\eta(x, y)), \quad \exists x^{*} \in \Phi(x), \forall x, y \in X .
\end{aligned}
$$

(ii) Strictly $\eta$-quasimonotone if there exist $\eta: K \times K \rightarrow X$ such that

$$
\begin{aligned}
& \langle F(y), \eta(x, y)\rangle>0, \quad \exists y^{*} \in \Phi(y) \\
& \Rightarrow \quad\langle F(x), \eta(x, y)\rangle>0, \quad \exists x^{*} \in \Phi(x), \forall x, y \in X .
\end{aligned}
$$

Example 2.3 If $F:(-\infty, 0] \rightarrow[0,+\infty)$ define by $F(x)=x^{2}$ and

$$
\eta(x, y)=|x-y| \quad \forall x, y \in(-\infty, 0]
$$

where $c>0$, then the mapping $F$ is a relaxed $\eta-\alpha$ pseudomonotone mapping with

$$
\alpha(z)= \begin{cases}-[|z|], & z>0, \\ {[|z|],} & z \leq 0 .\end{cases}
$$

But it is not a relaxed $\alpha$-pseudomonotone mapping. In fact, if we let $x=-1, y=0,\langle F(y), x-$ $y\rangle \geq 0$, but $\langle F(x), x-y\rangle<\alpha(x-y)$, which is a contradiction.

Example 2.4 If $F:(-\infty, 1) \rightarrow \mathbb{R}$ define by $F(x)=x^{2}-1$ and

$$
\eta(x, y)=-c(x-y) \quad \forall x, y \in(-\infty, 1)
$$

where $c>0$. Then the mapping $F$ is strictly $\eta$-quasimonotone but fails to be strictly quasimonotone since if $x \in(-1,1)$ and $y<-1$, then we have $\langle F(y), x-y\rangle \geq 0$ but $\langle F(x), x-y\rangle<0$. 
Definition 2.5 ([20]) Let $F: K \rightarrow X^{*}$ and $\eta: K \times K \rightarrow X$ be two mappings. $F$ is said to be $\eta$-hemicontinuous if, for any fixed $x, y \in K$, the mapping $f:[0,1] \rightarrow(-\infty,+\infty)$ defined by $f(t)=\langle F(x+t(y-x)), \eta(y, x)\rangle$ is continuous at $0^{+}$.

If $\eta(x, y)=x-y \forall x, y \in K$, then $F$ is said to be hemicontinuous.

Definition 2.6 ([29]) A mapping $F: K \rightarrow 2^{X}$ is said to be a KKM mapping if, for any $\left\{x_{1}, \ldots, x_{n}\right\} \subset K, \operatorname{co}\left\{x_{1}, \ldots, x_{n}\right\} \subset \bigcup_{i=1}^{n} F\left(x_{i}\right)$, where $\operatorname{co}\left\{x_{1}, \ldots, x_{n}\right\}$ denotes the convex hull of $x_{1}, \ldots, x_{n}$.

Lemma 2.7 ([29]) Let $K$ be a nonempty subset of a Hausdorff topological vector space $X$ and let $F: K \rightarrow 2^{X}$ be a KKM mapping. If $F(x)$ is closed in $X$ for every $x$ in $K$ and compact for some $x_{0} \in K$, then

$$
\bigcap_{x \in K} F(x) \neq \emptyset
$$

Lemma 2.8 (Michael selection theorem [30]) Let $X$ be a paracompact space and $Y$ be a Banach space. Then every lower semicontinuous multivalued mapping from $X$ to the family of nonempty, closed, convex subsets of $Y$ admits a continuous selection.

\section{Generalized variational-like inequality with relaxed $\eta-\alpha$ pseudomonotone mappings}

In this section, we will discuss the existence of solutions for the following variational-like inequality and generalized variational-like inequality with relaxed $\eta-\alpha$ pseudomonotone mappings.

Theorem 3.1 Let $K$ be a nonempty closed convex subset of a real reflexive Banach space $X$. Let $F: K \rightarrow X^{*}$ and $\eta: K \times K \rightarrow X$ be mappings. Assume that:

(i) $F$ is an $\eta$-hemicontinuous and relaxed $\eta$ - $\alpha$ pseudomonotone;

(ii) $\eta(x, x)=0$ for all $x \in K$;

(iii) $\eta(t x+(1-t) z, y)=t \eta(x, y)+(1-t) \eta(z, y)$ for all $x, y, z \in K, t \in[0,1]$.

Then $x \in K$ is a solution of $\operatorname{VLI}(K, F)$ if and only if

$$
\langle F(y), \eta(y, x)\rangle \geq \alpha(\eta(y, x)) \quad \forall y \in K .
$$

Proof Suppose that $x \in K$ is a solution of $\operatorname{VLI}(K, F)$. Since $F$ is relaxed $\eta$ - $\alpha$ pseudomonotone, we have

$$
\langle F(y), \eta(y, x)| \geq \alpha(\eta(y, x)) \quad \forall y \in K,
$$

and hence $x \in K$ is a solution of (3.1). Conversely, suppose that $x \in K$ is a solution of (3.1) and $y \in K$ be any point. Letting $x_{t}=t y+(1-t) x, t \in(0,1]$, we have $x_{t} \in K$. It follows from (3.1) that

$$
\left\langle F\left(x_{t}\right), \eta\left(x_{t}, x\right)\right| \geq \alpha\left(\eta\left(x_{t}, x\right)\right) .
$$


By the conditions of $\eta$, we have

$$
\begin{aligned}
\left\langle F\left(x_{t}\right), \eta\left(x_{t}, x\right)\right\rangle & =\left\langle F\left(x_{t}\right), \eta(t y+(1-t) x, x)\right\rangle \\
& =t\left\langle F\left(x_{t}\right), \eta(y, x)\right\rangle+(1-t)\left\langle F\left(x_{t}\right), \eta(x, x)\right\rangle \\
& =t\left\langle F\left(x_{t}\right), \eta(y, x)\right\rangle .
\end{aligned}
$$

It follows from (3.2) and (3.3) that

$$
t\left\langle F\left(x_{t}\right), \eta(y, x)\right\rangle=\left\langle F\left(x_{t}\right), \eta\left(x_{t}, x\right)\right\rangle \geq \alpha\left(\eta\left(x_{t}, x\right)\right) \quad \forall t \in(0,1] .
$$

So, we have

$$
\left\langle F\left(x_{t}\right), \eta(y, x)\right| \geq \frac{\alpha\left(\eta\left(x_{t}, x\right)\right)}{t}=\frac{\alpha(\operatorname{t\eta }(y, x))}{t} \quad \forall t \in(0,1] .
$$

Letting $t \rightarrow 0^{+}$, we get

$$
\langle F(x), \eta(y, x)\rangle \geq 0 \quad \forall y \in K
$$

Theorem 3.2 Let $X$ be a real reflexive Banach space and $K \subset X$ be a closed convex set. Let $F: K \rightarrow X^{*}$ and $\eta: K \times K \rightarrow X$ be are mappings. Assume that:

(i) $F$ is a relaxed $\eta$ - $\alpha$ pseudomonotone mapping and $\eta$-hemicontinuous;

(ii) $\eta(x, x)=0$ for all $x \in K$;

(iii) $\eta(t x+(1-t) z, y)=t \eta(x, y)+(1-t) \eta(z, y)$ for all $x, y, z \in K, t \in[0,1]$ and $\eta$ is lower semicontinuous;

(iv) $\alpha: X \rightarrow \mathbb{R}$ is lower semicontinuous.

Then the following statements are equivalent:

(a) There exists a reference point $x^{\mathrm{ref}} \in K$ such that the set

$$
L_{<}\left(F, x^{\mathrm{ref}}\right):=\left\{x \in K:\left\langle F(x), \eta\left(x, x^{\mathrm{ref}}\right)\right\rangle<\alpha\left(\eta\left(x, x^{\mathrm{ref}}\right)\right)\right\}
$$

is bounded (possibly empty).

(b) The variational-like inequality $\operatorname{VLI}(K, F)$ has a solution.

Moreover, if there exists a vector $x^{\mathrm{ref}} \in K$ such that the set

$$
L_{\leq}\left(F, x^{\mathrm{ref}}\right):=\left\{x \in K:\left\langle F(x), \eta\left(x, x^{\mathrm{ref}}\right)\right\rangle \leq \alpha\left(\eta\left(x, x^{\mathrm{ref}}\right)\right)\right\}
$$

is bounded and $\eta(x, y)+\eta(y, x)=0$ for all $x, y$ in $K$, then the solution set $\operatorname{SOL}(K, F)$ is nonempty and bounded.

Proof Suppose that there exists a reference point $x^{\text {ref }} \in K$, which satisfies (a). Then there exists an open ball, denoted by $\Omega$ such that

$$
L_{<}\left(F, x^{\mathrm{ref}}\right) \cup\left\{x^{\mathrm{ref}}\right\} \subset \Omega
$$

We combine this with the obvious property $\partial \Omega \cap L_{<}\left(F, x^{\mathrm{ref}}\right)=\emptyset$. Thus $\left\langle F(x), \eta\left(x, x^{\mathrm{ref}}\right)\right\rangle \geq$ $\alpha\left(\eta\left(x, x^{\mathrm{ref}}\right)\right) \forall x \in K \cap \partial \Omega$. Define the set-valued mappings $T, S: K \rightarrow 2^{X}$, for any $x \in K$, by

$$
T(x)=\{y \in K \cap \bar{\Omega}:\langle F(y), \eta(x, y)\rangle \geq 0\}
$$


and

$$
S(x)=\{y \in K \cap \bar{\Omega}:\langle F(x), \eta(x, y)\rangle \geq \alpha(\eta(x, y))\}
$$

We claim that $T$ is a KKM mapping. Indeed, if $T$ is not a KKM mapping, then there exists $\left\{x_{1}, x_{2}, \ldots, x_{n}\right\} \subset K$ such that $\operatorname{co}\left\{x_{1}, x_{2}, \ldots, x_{n}\right\} \nsubseteq \bigcup_{i=1}^{n} T\left(x_{i}\right)$. That is, there exists a $x_{0} \in$ $\operatorname{co}\left\{x_{1}, x_{2}, \ldots, x_{n}\right\}, x_{0}=\sum_{i=1}^{n} t_{i} x_{i}$, where $t_{i} \geq 0, i=1,2, \ldots, n, \sum_{i=1}^{n} t_{i}=1$, but $x_{0} \notin \bigcup_{i=1}^{n} T\left(x_{i}\right)$. By the definition of $T$, we have

$$
\left\langle F\left(x_{0}\right), \eta\left(x_{i}, x_{0}\right)\right\rangle<0, \quad i=1,2, \ldots, n .
$$

Since $\sum_{i=1}^{n} t_{i}=1$ for $t_{i} \geq 0(i=1,2, \ldots, n)$, it follows that

$$
\sum_{i=1}^{n} t_{i}\left\langle F\left(x_{0}\right), \eta\left(x_{i}, x_{0}\right)\right\rangle<0 .
$$

On the other hand, we note that

$$
\begin{aligned}
\sum_{i=1}^{n} t_{i}\left\langle F\left(x_{0}\right), \eta\left(x_{i}, x_{0}\right)\right\rangle & =\left\langle F\left(x_{0}\right), \eta\left(\sum_{i=1}^{n} t_{i} x_{i}, x_{0}\right)\right\rangle \\
& =\left\langle F\left(x_{0}\right), \eta\left(x_{0}, x_{0}\right)\right\rangle \\
& =0 .
\end{aligned}
$$

It is a contradiction and this implies that $T$ is a KKM mapping. Now we show that $T(x) \subset$ $S(x)$ for all $x \in K$. For any given $x \in K$, let $y \in T(x)$. Thus, we have $\langle F(y), \eta(x, y)\rangle \geq 0$. Since $F$ is a relaxed $\eta$ - $\alpha$ pseudomonotone, we obtain $\langle F(x), \eta(x, y)\rangle \geq \alpha(\eta(x, y))$. This implies that $y \in S(x)$ and so $T(x) \subset S(x)$ for all $x \in K$. It follows that $S$ is also a KKM mapping.

From the assumptions, we know that $S(x)$ is weakly closed. In fact, since $\eta$ and $\alpha$ are lower semicontinuous, we see that $S(x)$ is a weakly closed subset of $K \cap \bar{\Omega}$. Since $K \cap$ $\bar{\Omega}$ is a weakly compact and $S(x)$ is a weakly closed subset of $K \cap \bar{\Omega}$, we see that $S(x)$ is weakly compact for each $x \in K$. Thus, the conditions of Lemma 2.7 are satisfied in the weak topology. By Lemma 2.7 and Theorem 3.1, we have

$$
\bigcap_{x \in K} T(x)=\bigcap_{x \in K} S(x) \neq \emptyset .
$$

It follows that there exists $z \in K \cap \bar{\Omega}$ such that

$$
\langle F(z), \eta(x, z)\rangle \geq 0 \quad \forall x \in K
$$

Hence $z \in \operatorname{SOL}(K, F)$.

Assume that (b) holds. We take any $x^{\text {ref }} \in \operatorname{SOL}(K, F)$. That is,

$$
\left\langle F\left(x^{\mathrm{ref}}\right), \eta\left(x, x^{\mathrm{ref}}\right)\right\rangle \geq 0 \quad \forall x \in K
$$


By the relaxed $\eta-\alpha$ pseudomonotonicity of $F$, we have

$$
\left\langle F(x), \eta\left(x, x^{\mathrm{ref}}\right)\right\rangle \geq \alpha\left(\eta\left(x, x^{\mathrm{ref}}\right)\right) \quad \forall x \in K .
$$

Hence $L_{<}\left(F, x^{\mathrm{ref}}\right)=\emptyset$ and (a) is valid.

Finally, suppose that there is some $x^{\text {ref }} \in K$ such that the set $L_{\leq}\left(F, x^{\text {ref }}\right)$ is bounded. Then $\operatorname{SOL}(K, F)$ is nonempty by virtue of the implication (a) $\Rightarrow$ (b). To prove that $\operatorname{SOL}(K, F)$ is bounded, it suffices to show that $\operatorname{SOL}(K, F) \subset L_{\leq}\left(F, x^{\text {ref }}\right)$. Assume that $x \in \operatorname{SOL}(K, F)$, but $x \notin L_{\leq}\left(F, x^{\text {ref }}\right)$. Thus, we have

$$
\langle F(x), \eta(y, x)\rangle \geq 0 \quad \forall y \in K
$$

and

$$
\left\langle F(x), \eta\left(x, x^{\mathrm{ref}}\right)\right\rangle>\alpha\left(\eta\left(x, x^{\mathrm{ref}}\right)\right) .
$$

Substituting $y=x^{\text {ref }}$ into the inequality in (3.4), we have

$$
\left\langle F(x), \eta\left(x^{\mathrm{ref}}, x\right)\right\rangle \geq 0
$$

This implies that $\left\langle F(x), \eta\left(x, x^{\mathrm{ref}}\right)\right\rangle \leq 0$. From (3.5) and (3.6), we have $\alpha\left(\eta\left(x, x^{\mathrm{ref}}\right)\right)<0$. By (3.6) and $F$ is relaxed $\eta-\alpha$ pseudomonotone, we obtain

$$
\left\langle F\left(x^{\mathrm{ref}}\right), \eta\left(x^{\mathrm{ref}}, x\right)\right\rangle \geq \alpha\left(\eta\left(x^{\mathrm{ref}}, x\right)\right) .
$$

It implies that $\left\langle F\left(x^{\mathrm{ref}}\right), \eta\left(x, x^{\mathrm{ref}}\right)\right\rangle \leq \alpha\left(\eta\left(x, x^{\mathrm{ref}}\right)\right)$. By Theorem 3.1 and (3.5), we get $\left\langle F\left(x^{\mathrm{ref}}\right)\right.$, $\left.\eta\left(x, x^{\text {ref }}\right)\right\rangle \geq 0$. Hence

$$
0 \leq\left\langle F\left(x^{\mathrm{ref}}\right), \eta\left(x, x^{\mathrm{ref}}\right)\right\rangle \leq \alpha\left(\eta\left(x, x^{\mathrm{ref}}\right)\right)<0 .
$$

It is a contradiction. Therefore $x \in L_{\leq}\left(F, x^{\mathrm{ref}}\right)$.

Theorem 3.3 Let $X$ be a real reflexive Banach space and $K \subset X$ be a closed convex set. Let $\Phi: K \rightarrow 2^{X^{*}}$ and $\eta: K \times K \rightarrow X$ be are mappings. Assume that:

(i) $\Phi$ is a lower semicontinuous multifunction with nonempty closed convex values, where $X^{*}$ is endowed with the norm topology;

(ii) $\Phi$ is a relaxed $\eta$ - $\alpha$ pseudomonotone mapping;

(iii) $\eta(x, x)=0$ for all $x \in K$;

(iv) $\eta(t x+(1-t) z, y)=t \eta(x, y)+(1-t) \eta(z, y)$ for all $x, y, z \in K, t \in[0,1]$ and $\eta$ is lower semicontinuous;

(v) $\alpha: X \rightarrow \mathbb{R}$ is lower semicontinuous.

Then the following statements are equivalent:

(a) There exists a reference point $x^{\text {ref }} \in K$ such that the set

$$
L_{<}\left(\Phi, x^{\mathrm{ref}}\right):=\left\{x \in K: \inf _{x^{*} \in \Phi(x)}\left\langle x^{*}, \eta\left(x, x^{\mathrm{ref}}\right)\right\rangle<\alpha\left(\eta\left(x, x^{\mathrm{ref}}\right)\right)\right\}
$$

is bounded (possibly empty).

(b) The generalized variational-like inequality $\operatorname{GVLI}(K, \Phi)$ has a solution. 
Proof Since $\Phi$ is lower semicontinuous multifunction with nonempty closed convex values, by Michael's selection theorem (see for instance [30]) it admits a continuous selection; that is, there exists a continuous mapping $F: K \rightarrow X^{*}$ such that $F(x) \in \Phi(x)$ for every $x \in K$. If (a) holds, then there exists an open ball, denoted by $\Omega$ such that

$$
L_{<}\left(\Phi, x^{\mathrm{ref}}\right) \cup\left\{x^{\mathrm{ref}}\right\} \subset \Omega
$$

We combine this with the obvious property $\partial \Omega \cap L_{<}\left(\Phi, x^{\text {ref }}\right)=\emptyset$. Thus, we have

$$
\left\langle F(x), \eta\left(x, x^{\mathrm{ref}}\right)\right\rangle \geq \inf _{x^{*} \in \Phi(x)}\left\langle x^{*}, \eta\left(x, x^{\mathrm{ref}}\right)\right\rangle \geq \alpha\left(\eta\left(x, x^{\mathrm{ref}}\right)\right) \quad \forall x \in K \cap \partial \Omega
$$

Applying Theorem 3.2, we get $\operatorname{SOL}(K, F) \neq \emptyset$. For any $x \in \operatorname{SOL}(K, F)$, if we choose $x^{*}=F(x)$ then

$$
\left\langle x^{*}, \eta(y, x)\right\rangle \geq 0 \quad \forall y \in K \text {. }
$$

It follows that $\emptyset \neq \operatorname{SOL}(K, F) \subset \operatorname{SOL}(K, \Phi)$.

We prove that (b) $\Rightarrow\left(\right.$ a). Assume that (b) holds. We take any $x^{\text {ref }} \in \operatorname{SOL}(K, \Phi)$. Thus there exists $x^{*} \in \Phi\left(x^{\text {ref }}\right)$ satisfying

$$
\left\langle x^{*}, \eta\left(y, x^{\mathrm{ref}}\right)\right\rangle \geq 0 \quad \forall y \in K
$$

Because $\Phi$ is a relaxed $\eta-\alpha$ pseudomonotone, we obtain

$$
\left\langle y^{*}, \eta\left(y, x^{\mathrm{ref}}\right)\right\rangle \geq \alpha\left(\eta\left(y, x^{\mathrm{ref}}\right)\right) \quad \forall y \in K, y^{*} \in \Phi(y) .
$$

It follows that

$$
\inf _{y^{*} \in \Phi(y)}\left\langle y^{*}, \eta\left(y, x^{\mathrm{ref}}\right)\right\rangle \geq \alpha\left(\eta\left(y, x^{\mathrm{ref}}\right)\right) \quad \forall y \in K \text {. }
$$

Hence $L_{<}\left(\Phi, x^{\mathrm{ref}}\right)=\emptyset$ and (a) is valid.

\section{Generalized variational-like inequality with strictly $\eta$-quasimonotone mappings}

In this section, we will discuss the existence of solutions for the following variationallike inequality and generalized variational-like inequality with strictly $\eta$-quasimonotone mappings.

Theorem 4.1 Let $K$ be a nonempty closed convex subset of a real reflexive Banach space $X$.

Let $F: K \rightarrow X^{*}$ and $\eta: K \times K \rightarrow X$ be mappings. Assume that:

(i) $F$ is $\eta$-hemicontinuous and strictly $\eta$-quasimonotone;

(ii) $\eta(x, x)=0$ for all $x \in K$;

(iii) $\eta(x, y)+\eta(y, x)=0$ for all $x, y \in K$;

(iv) for any fixed $y, z \in K$, the mapping $x \mapsto\langle T z, \eta(x, y)\rangle$ is convex.

Then $x \in K$ is a solution of $\operatorname{VLI}(K, F)$ if and only if

$$
\langle F(y), \eta(y, x)\rangle \geq 0 \quad \forall y \in K
$$


Proof Suppose that $x \in K$ is a solution of $\operatorname{VLI}(K, F)$. That is $\langle F(x), \eta(y, x)\rangle \geq 0 \forall y \in K$. To show that $\langle F(y), \eta(y, x)\rangle \geq 0 \forall y \in K$. Assume that there exists $y_{0} \in K$ such that $\left\langle F\left(y_{0}\right), \eta\left(y_{0}, x\right)\right\rangle<0$. By the property of $\eta$, we have $\left\langle F\left(y_{0}\right), \eta\left(x, y_{0}\right)\right\rangle>0$. Since $F$ is strictly $\eta$-quasimonotone, we have $\left\langle F(x), \eta\left(x, y_{0}\right)\right\rangle>0$. By the property of $\eta$ again, we get $\langle F(x)$, $\left.\eta\left(y_{0}, x\right)\right\rangle<0$. It is a contradiction. Hence $\langle F(y), \eta(y, x)\rangle \geq 0 \forall y \in K$.

Conversely, suppose that $x \in K$ is a solution of (4.1) and $y \in K$ is arbitrary. Letting $x_{t}=$ $t y+(1-t) x, t \in(0,1]$, we have $x_{t} \in K$. It follows from (4.1) that

$$
\left\langle F\left(x_{t}\right), \eta\left(x_{t}, x\right)\right\rangle \geq 0
$$

By assumption, we have

$$
\begin{aligned}
\left\langle F\left(x_{t}\right), \eta\left(x_{t}, x\right)\right\rangle & =\left\langle F\left(x_{t}\right), \eta(t y+(1-t) x, x)\right\rangle \\
& \leq t\left\langle F\left(x_{t}\right), \eta(y, x)\right\rangle+(1-t)\left\langle F\left(x_{t}\right), \eta(x, x)\right\rangle \\
& =t\left\langle F\left(x_{t}\right), \eta(y, x)\right\rangle .
\end{aligned}
$$

It follows from (4.2) and (4.3) that

$$
\left\langle F\left(x_{t}\right), \eta(y, x)\right\rangle \geq 0 \quad \forall t \in(0,1] .
$$

Since $F$ is $\eta$-hemicontinuous and letting $t \rightarrow 0^{+}$, we get

$$
\langle F(x), \eta(y, x)\rangle \geq 0 \quad \forall y \in K .
$$

Theorem 4.2 Let $X$ be a real reflexive Banach space and $K \subset X$ be a closed convex set. Let $F: K \rightarrow X^{*}$ and $\eta: K \times K \rightarrow X$ be are mappings. Assume that:

(i) $F$ is a strictly $\eta$-quasimonotone mapping and $\eta$-hemicontinuous;

(ii) $\eta(x, x)=0$ for all $x \in K$;

(iii) $\eta(x, y)+\eta(y, x)=0$ for all $x, y \in K$;

(iv) for any fixed $y, z \in K$, the mapping $x \mapsto\langle T z, \eta(x, y)\rangle$ is convex and $\eta$ is lower semicontinuous.

Then the following statements are equivalent:

(a) There exists a reference point $x^{\mathrm{ref}} \in K$ such that the set

$$
L_{<}\left(F, x^{\mathrm{ref}}\right):=\left\{x \in K:\left\langle F(x), \eta\left(x, x^{\mathrm{ref}}\right)\right\rangle<0\right\}
$$

is bounded (possibly empty).

(b) The variational-like inequality $\operatorname{VLI}(K, F)$ has a solution.

Moreover, if there exists a vector $x^{\mathrm{ref}} \in K$ such that the set

$$
L_{\leq}\left(F, x^{\mathrm{ref}}\right):=\left\{x \in K:\left\langle F(x), \eta\left(x, x^{\mathrm{ref}}\right)\right\rangle \leq 0\right\}
$$

is bounded, then the solution set $\mathrm{SOL}(K, F)$ is nonempty and bounded.

Proof Suppose that (a) holds. Then there exists a reference point $x^{\mathrm{ref}} \in K$ and an open ball, denoted by $\Omega$ such that

$$
L_{<}\left(F, x^{\mathrm{ref}}\right) \cup\left\{x^{\mathrm{ref}}\right\} \subset \Omega .
$$


We combine this with the obvious property $\partial \Omega \cap L_{<}\left(F, x^{\text {ref }}\right)=\emptyset$. Thus $\left\langle F(x), \eta\left(x, x^{\text {ref }}\right)\right\rangle \geq$ $\alpha\left(\eta\left(x, x^{\mathrm{ref}}\right)\right) \forall x \in K \cap \partial \Omega$. Defined the set-valued mappings $T, S: K \rightarrow 2^{X}$, for any $x \in K$, by

$$
T(x)=\{y \in K \cap \bar{\Omega}:\langle F(y), \eta(x, y)\rangle \geq 0\}
$$

and

$$
S(x)=\{y \in K \cap \bar{\Omega}:\langle F(x), \eta(x, y)| \geq 0\} .
$$

Since $\eta$ is lower semicontinuous, we find that $T(x)$ and $S(x)$ are weakly closed subsets of $K \cap \bar{\Omega}$. We claim that $T$ is a KKM mapping. Similar to the proof of Theorem 3.2 we show that $T$ is a KKM mapping. Now we show that $T(x) \subset S(x)$ for all $x \in K$. For any given $x \in K$, we let $y \in T(x)$. That is, $\langle F(y), \eta(x, y)\rangle \geq 0$. Since $F$ is strictly $\eta$-quasimonotone, we have $\langle F(x), \eta(x, y)\rangle \geq 0$. This implies that $y \in S(x)$ and so $T(x) \subset S(x)$ for all $x \in K$. It follows that $S$ is also a KKM mapping. Since $K \cap \bar{\Omega}$ is weakly compact and $S(x)$ is a weakly closed subset of $K \cap \bar{\Omega}$, we find that $S(x)$ is weakly compact for each $x \in K$. Thus, the condition of Lemma 2.7 is satisfied in the weak topology. By Lemma 2.7 and Theorem 4.1, we have

$$
\bigcap_{x \in K} T(x)=\bigcap_{x \in K} S(x) \neq \emptyset
$$

It follows that there exists $z \in K \cap \bar{\Omega}$ such that

$$
\langle F(z), \eta(x, z)\rangle \geq 0 \quad \forall x \in K .
$$

Hence $z \in \operatorname{SOL}(K, F)$.

Assume that (b) holds. We take any $x^{\text {ref }} \in \operatorname{SOL}(K, F)$, that is,

$$
\left\langle F\left(x^{\mathrm{ref}}\right), \eta\left(x, x^{\mathrm{ref}}\right)\right\rangle \geq 0 \quad \forall x \in K .
$$

By the strict $\eta$-quasimonotonicity of $F$, we have

$$
\left\langle F(x), \eta\left(x, x^{\mathrm{ref}}\right)\right| \geq 0 \quad \forall x \in K .
$$

Hence $L_{<}\left(F, x^{\text {ref }}\right)=\emptyset$ and (a) is valid.

Finally, suppose that there is some $x^{\text {ref }} \in K$ such that the set $L_{\leq}\left(F, x^{\text {ref }}\right)$ is bounded. Then $\operatorname{SOL}(K, F)$ is nonempty by virtue of the implication (a) $\Rightarrow$ (b). To prove that $\operatorname{SOL}(K, F)$ is bounded, it suffices to show that $\operatorname{SOL}(K, F) \subset L_{\leq}\left(F, x^{\text {ref }}\right)$. Assume that $x \in \operatorname{SOL}(K, F)$. Thus, we have

$$
\langle F(x), \eta(y, x)\rangle \geq 0 \quad \forall y \in K .
$$

Substituting $y=x^{\text {ref }}$ into the inequality in (4.4), we have

$$
\left\langle F(x), \eta\left(x^{\mathrm{ref}}, x\right)\right\rangle \geq 0
$$

This implies that $\left\langle F(x), \eta\left(x, x^{\mathrm{ref}}\right)\right\rangle \leq 0$. Therefore $x \in L_{\leq}\left(F, x^{\mathrm{ref}}\right)$. 
Theorem 4.3 Let $X$ be a real reflexive Banach space and $K \subset X$ be a closed convex set. Let $\Phi: K \rightarrow 2^{X^{*}}$ and $\eta: K \times K \rightarrow X$ be are mappings. Assume that:

(i) $\Phi$ is a lower semicontinuous multifunction with nonempty closed convex values, where $X^{*}$ is endowed with the norm topology;

(ii) $\Phi$ is a strictly $\eta$-quasimonotone mapping;

(iii) $\eta(x, x)=0$ for all $x \in K$;

(iv) $\eta(x, y)+\eta(y, x)=0$ for all $x, y \in K$;

(v) for any fixed $y, z \in K$, the mapping $x \mapsto\langle T z, \eta(x, y)\rangle$ is convex and $\eta$ is lower semicontinuous.

Then the following statements are equivalent:

(a) There exists a reference point $x^{\mathrm{ref}} \in K$ such that the set

$$
L_{<}\left(\Phi, x^{\mathrm{ref}}\right):=\left\{x \in K: \inf _{x^{*} \in \Phi(x)}\left\langle x^{*}, \eta\left(x, x^{\mathrm{ref}}\right)\right\rangle<0\right\}
$$

is bounded (possibly empty).

(b) The generalized variational-like inequality $\operatorname{GVLI}(K, \Phi)$ has a solution.

Proof Since $\Phi$ is a lower semicontinuous multifunction with nonempty closed convex values, by Michael's selection theorem (see for instance [30]) it admits a continuous selection; that is, there exists a continuous mapping $F: K \rightarrow X^{*}$ such that $F(x) \in \Phi(x)$ for every $x \in K$. If (a) holds, then there exists an open ball, denoted by $\Omega$, such that

$$
L_{<}\left(\Phi, x^{\mathrm{ref}}\right) \cup\left\{x^{\mathrm{ref}}\right\} \subset \Omega
$$

We combine this with the obvious property $\partial \Omega \cap L_{<}\left(\Phi, x^{\text {ref }}\right)=\emptyset$. Then we have

$$
\left\langle F(x), \eta\left(x, x^{\mathrm{ref}}\right)\right| \geq \inf _{x^{*} \in \Phi(x)}\left\langle x^{*}, \eta\left(x, x^{\mathrm{ref}}\right)\right\rangle \geq 0 \quad \forall x \in K \cap \partial \Omega
$$

Applying Theorem 4.2 , we get $\operatorname{SOL}(K, F) \neq \emptyset$. For any $x \in \operatorname{SOL}(K, F)$, if we choose $x^{*}=F(x)$ then

$$
\left\langle x^{*}, \eta(y, x)\right\rangle \geq 0 \quad \forall y \in K
$$

It follows that $\emptyset \neq \operatorname{SOL}(K, F) \subset \operatorname{SOL}(K, \Phi)$.

We prove that (b) $\Rightarrow$ (a). Assume that (b) holds. We take any $x^{\text {ref }} \in \operatorname{SOL}(K, \Phi)$. Thus there exists $x^{*} \in \Phi\left(x^{\mathrm{ref}}\right)$ satisfying

$$
\left\langle x^{*}, \eta\left(y, x^{\text {ref }}\right)\right| \geq 0 \quad \forall y \in K .
$$

Because $\Phi$ is strictly $\eta$-quasimonotone and Theorem 4.1, we obtain

$$
\left\langle y^{*}, \eta\left(y, x^{\text {ref }}\right)\right\rangle \geq 0 \quad \forall y \in K, y^{*} \in \Phi(y) .
$$

It follows that

$$
\inf _{y^{*} \in \Phi(y)}\left\langle y^{*}, \eta\left(y, x^{\mathrm{ref}}\right)\right\rangle \geq 0 \quad \forall y \in K
$$

Hence $L_{<}\left(\Phi, x^{\mathrm{ref}}\right)=\emptyset$ and (a) is valid. 


\section{Competing interests}

The authors declare that they have no competing interests.

\section{Authors' contributions}

The work presented here was carried out in collaboration between all authors. SP, C-FW and AA defined the research theme. SP and C-FW designed theorems and methods of proof and interpreted the results. AA proved the theorems, interpreted the results and wrote the paper. All authors read and approved the final manuscript.

\section{Author details}

'Department of Mathematics, Faculty of Science, Naresuan University, Phitsanulok, 65000, Thailand. ${ }^{2}$ Center for Fundamental Science, Kaohsiung Medical University, Kaohsiung, 807, Taiwan.

\section{Acknowledgements}

The first author would like to thank the Thailand Research Fund for financial support and the second author is also supported by the Royal Golden Jubilee Program under Grant PHD/0282/2550, Thailand.

\section{Received: 23 April 2014 Accepted: 20 October 2014 Published: 04 Nov 2014}

\section{References}

1. Giannessi, F: Theorems of alternative, quadratic programs and complementarity problems. In: Cottle, RW, Giannessi, F, Lions, JL (eds.) Variational Inequalities and Complementarity Problems, pp. 151-186. Wiley, New York (1980)

2. Hartmann, P, Stampacchia, G: On some nonlinear elliptic differential functional equations. Acta Math. 115, 153-188 (1966)

3. Kinderlehrer, D, Stampacchia, G: An Introduction to Variational Inequalities and Their Applications. Academic Press, New York (1980)

4. Aussel, D, Hadjisavvas, N: On quasimonotone variational inequalities. J. Optim. Theory Appl. 121, 445-450 (2004)

5. Bianchi, M, Hadjisavvas, N, Shaible, S: Minimal coercivity conditions and exceptional families of elements in quasimonotone variational inequalities. J. Optim. Theory Appl. 122, 1-17 (2004)

6. Crouzeix, JP: Pseudomonotone variational inequality problems: existence of solutions. Math. Program. 78, 305-314 (1997)

7. Daniilidis, A, Hadjisavvas, N: Coercivity conditions and variational inequalities. Math. Program. 86, 433-438 (1999)

8. Konnov, IV: Generalized monotone equilibrium problems and variational inequalities. In: Hadjisavvas, N, Komlósi, S, Schaible, S (eds.) Handbook of Generalized Convexity and Generalized Monotonicity, pp. 559-618. Springer, Berlin (2005)

9. Yao, JC: Variational inequalities with generalized monotone operators. Math. Oper. Res. 19, 691-705 (1994)

10. Yao, JC: Multi-valued variational inequalities with $K$-pseudomonotone operators. J. Optim. Theory Appl. 80, 63-74 (1994)

11. Yao, JC, Chadli, O: Pseudomonotone complementarity problems and variational inequalities. In: Hadjisavvas, N, Komlósi, S, Schaible, S (eds.) Handbook of Generalized Convexity and Generalized Monotonicity, pp. 501-558. Springer, Berlin (2005)

12. Ricceri, B: Basic existence theorems for generalized variational and quasi-variational inequalities. In: Giannessi, F, Maugeri, A (eds.) Variational Inequalities and Network Equilibrium Problems, pp. 251-255. Plenum, New York (1995)

13. Yen, ND: On a problem of B. Ricceri on variational inequalities. In: Cho, YJ, Kim, JK, Kang, SM (eds.) Fixed Point Theory and Applications, vol. 5, pp. 163-173. Nova Science Publishers, New York (2004)

14. Chang, SS, Lee, BS, Chen, YQ: Variational inequalities for monotone operators in nonreflexive Banach spaces. Appl. Math. Lett. 8, 29-34 (1995)

15. Verma, RU: On generalized variational inequalities involving relaxed Lipschitz and relaxed monotone operators. J. Math. Anal. Appl. 213, 387-392 (1997)

16. Fang, YP, Huang, NJ: Existence results for systems of strong implicit vector variational inequalities. Acta Math. Hung 103, 265-277 (2004)

17. Facchinei, F, Pang, JS: Finite-Dimensional Variational Inequalities and Complementarity Problems, vol. I. Springer, Berlin (2003)

18. Facchinei, F, Pang, JS: Finite-Dimensional Variational Inequalities and Complementarity Problems, vol. II. Springer, Berlin (2003)

19. Kien, BT, Yao, JC, Yen, ND: On the solution existence of pseudomonotone variational inequalities. J. Glob. Optim. 41, 135-145 (2008)

20. Bai, MR, Zhoua, SZ, Ni, GY: Variational-like inequalities with relaxed $\eta$ - $\alpha$ pseudomonotone mappings in Banach spaces. Appl. Math. Lett. 19, 547-554 (2006)

21. $\mathrm{Wu}, \mathrm{KQ}$, Huang, NJ: Vector variational-like inequalities with relaxed $\eta$ - $\alpha$ pseudomonotone mappings in Banach spaces. J. Math. Inequal. 1, 281-290 (2007)

22. Pourbarat, $\mathrm{K}$, Abbasi, $\mathrm{M}$ : On the vector variational-like inequalities with relaxed $\eta$ - $\alpha$ pseudomonotone mappings. Iran. J. Math. Sci. Inform. 4(1), 37-42 (2009)

23. Goeleven, D, Motreanu, D: Eigenvalue and dynamic problems for variational and hemivariational inequalities, Commun. Appl. Nonlinear Anal. 3, 1-21 (1996)

24. Siddiqi, AH, Ansari, QH, Kazmi, KR: On nonlinear variational inequalities. Indian J. Pure Appl. Math. 25, 969-973 (1994)

25. Verma, RU: On monotone nonlinear variational inequalities problems. Comment. Math. Univ. Carol. 39, 91-98 (1998)

26. Cottle, RW, Yao, JC: Pseudomonotone complementarity problems in Hilbert spaces. J. Optim. Theory Appl. 78, 281-295 (1992)

27. Karamardian, S: Complementarity problems over cones with monotone and pseudomonotone maps. J. Optim. Theory Appl. 18, 445-454 (1976)

28. Ceng, LC, Lee, GM, Yao, JC: Generalized variational-like inequalities with compositely monotone multifunctions. J. Korean Math. Soc. 45(3), 841-858 (2008)

29. Yuan, GXZ: KKM Theory and Applications in Nonlinear Analysis. Dekker, New York (1999)

30. Michael, EA: Continuous selections, I. Ann. Math. 63, 361-382 (1956) 
10.1186/1029-242X-2014-442

Cite this article as: Arunchai et al.: Existence theorems for relaxed $\eta$ - $\alpha$ pseudomonotone and strictly

$\eta$-quasimonotone generalized variational-like inequalities. Journal of Inequalities and Applications 2014, 2014:442

Submit your manuscript to a SpringerOpen ${ }^{\circ}$ journal and benefit from:

- Convenient online submission

- Rigorous peer review

- Immediate publication on acceptance

Open access: articles freely available online

- High visibility within the field

- Retaining the copyright to your article

Submit your next manuscript at $>$ springeropen.com 